\title{
TAUBERIAN-TYPE THEOREMS WITH APPLICATION TO THE STIELTJES TRANSFORMATION
}

\author{
S. B. GAIKWAD AND M. S. CHAUDHARY
}

Received 22 November 2004; Revised 12 April 2006; Accepted 22 June 2006

In the first part, we define the space $L^{\prime}(r)$ and the modified Stieltjes transformation introduced by Lavoine and Misra (1979) and Marichev (1983), respectively. In the second part of the paper, we extend Tauberian-type theorems for the distributional Stieltjes transformations to the distributional modified Stieltjes transformations.

Copyright (c) 2006 Hindawi Publishing Corporation. All rights reserved.

\section{Introduction}

The Abelian and Tauberian-type theorems were introduced by Stanković [7] and Pilipović et al. [5]. In the first part of this paper, we give the definition of the quasiasymptotic expansion at $0^{+}$and the quasiasymptotic behaviour of distributions at infinity from $S_{+}^{\prime}$ introduced in [1]. In this paper, we give the definition of space $L^{\prime}(r)$, classical Stieltjes transformation, modified Stieltjes transformation, and generalized modified Stieltjes transformation. This enables us to obtain, in the second part of the paper, the Tauberiantype theorems of quasiasymptotic behaviour of distributions at infinity. We give sufficient conditions under which the behaviour at infinity of the modified Stieltjes transformation $\Gamma(r+1)\left(T_{r+1} f\right)(x), r \in \mathbb{R} \backslash(-\mathbb{N}), f \in L^{\prime}(r)$ determines the quasiasymptotic behaviour of $f$ at infinity.

Notation 1.1. As usually $\mathbb{R}, \mathbb{C}, \mathbb{N}$ are the spaces of real, complex, and natural numbers; $\mathbb{N}_{0}=\mathbb{N} \cup\{0\} . D$ is the space of infinitely differentiable functions with compact support. $S_{+}^{\prime}$ denotes the space of tempered distributions with support in the $[0, \infty)$. The space of rapidly decreasing functions is denoted as $S, S^{\prime}$ is the space of all distributions of slow growth. $T_{r+1}$ denotes the modified Stieltjes transformation with index $r$.

A positive continuous function $L$ defined on $(0, \infty)$ is called slowly varying function at $\infty$ if for every $k>0$,

$$
\lim _{k \rightarrow \infty} \frac{L(k x)}{L(k)}=1
$$

Hindawi Publishing Corporation International Journal of Mathematics and Mathematical Sciences Volume 2006, Article ID 75816, Pages 1-10

DOI 10.1155/IJMMS/2006/75816 
2 Tauberian-type theorems to Stieltjes transformations

We denote by $\sum_{\infty}$ the set of all slowly varying functions at $\infty$. For the properties of slowly varying functions, we refer the reader to [6].

If $L$ is a slowly varying function at $\infty$, then for every $\varepsilon>0$, there is $A_{\varepsilon}>0$ such that

$$
x^{-\varepsilon}<L(x)<x^{\varepsilon} \quad \text { when } x>A_{\varepsilon} .
$$

Recall that for $\alpha>-1, x_{+}^{\alpha}=H(x) x^{\alpha}$, where $H$ is Heaviside's function. The following scale of distributions from $S_{+}^{\prime}$ has been used in the investigations of the quasiasymptotic behaviour of distributions:

$$
f_{\alpha+1}= \begin{cases}\frac{H t^{\alpha}}{\Gamma(\alpha+1)}, & \alpha>-1, \\ D^{n} f_{\alpha+n+1}, & \alpha \leq-1, \alpha+n>-1,\end{cases}
$$

where $D$ is the distributional derivative.

\section{Definitions}

\subsection{Definition of the quasiasymptotic behaviour (q.a.b.)}

Definition 2.1. The quasiasymptotic behaviour of distribution (q.a.b.) at infinity.

If $T$ is a distribution from $S_{+}^{\prime}$ such that the distributional limit

$$
\lim _{k \rightarrow \infty} \frac{T(k x)}{\rho(k)}=\gamma(x)
$$

exists in $S^{\prime}(\gamma(x) \neq 0)$, then $T$ is called the quasiasymptotic behaviour at infinity related to the regular function $\rho(k)=k^{\alpha} L(k)$ with the limit $\gamma$; write this as

$$
T \stackrel{q}{\sim} \gamma \quad \text { in } S^{\prime} \text { as } x \longrightarrow \infty
$$

Here $\rho$ is regularly varying at infinity and the limit $\gamma$ from $S_{+}^{\prime}$ is of the form

$$
\gamma(x)=\mathbb{C} f_{\alpha+1}(x)
$$

We will repeat in this section some well-known facts about the quasiasymptotic behaviour from [8].

Let $f \in S_{+}^{\prime}$. It is said that $f$ has the q.a.b. at $\infty$ with the limit $g \neq 0$ with respect to

$$
\begin{gathered}
k^{\alpha} L(k), \quad L \in \sum_{\infty}\left(\left(\frac{1}{k}\right)^{\alpha} L\left(\frac{1}{k}\right), L \in \sum_{0}\right), \alpha \in \mathbb{R}, \\
\quad \text { if } \lim _{k \rightarrow \infty}\left\langle\frac{f(k t)}{k^{\alpha} L(k)}, \phi(t)\right\rangle=\langle g(t), \phi(t)\rangle, \quad \phi \in S .
\end{gathered}
$$


Definition 2.2. A function $\rho:(a, \infty) \rightarrow \mathbb{R}, a \in \mathbb{R}$, is called regularly varying function at infinity if it is positive, measurable, and there exists a real number $\alpha$ such that for each

$$
x>0, \quad \lim _{k \rightarrow \infty} \frac{\rho(k x)}{\rho(k)}=x^{\alpha} ;
$$

where the number $\alpha$ is called the index of $\rho$.

2.2. Space $L^{\prime}(r)$. We extend the definition of the space $I^{\prime}(r)$ given in [4] and using the same idea, we give the definition of space $L^{\prime}(r)$.

$L^{\prime}(r), r \in \mathbb{R} \backslash(-\mathbb{N})$, the space of all distributions $f \in S_{+}^{\prime}(\mathbb{R})$ such that there exist $k \in \mathbb{N}_{0}$ and locally integrable function $F, \operatorname{supp} F \subset[0, \infty)$, so that $f$ is of the form

$$
f=t^{-r} D^{k} F
$$

and there exist $C=C(F)$ and $\varepsilon=\varepsilon(F)>0$ such that

$$
|F(x)| \leq C(1+x)^{r+k-\varepsilon}, \quad x \geq 0 .
$$

The Stieltjes transformation $S_{r}(f)(s), r \in \mathbb{R} \backslash(-\mathbb{N})$, is complex-valued function, defined by

$$
S_{r}(f(t))(s)=\int_{0}^{\infty} \frac{f(t)}{(s+t)^{r+1}} d t \quad s \in \mathbb{C} \backslash(-\infty, 0], 0<t<\infty, r \in \mathbb{R} \backslash(-\mathbb{N}) .
$$

Modified Stieltjes transformation introduced by Marichev is defined as

$$
T_{\alpha}(f(x))=\frac{1}{\Gamma(\alpha)} \int_{0}^{\infty}\left(1+\frac{x}{y}\right)^{-\alpha} \cdot \frac{1}{y} f(y) d y, \quad x \in \mathbb{C} \backslash(-\infty, 0], 0<y<\infty, r \in \mathbb{R} \backslash(-\mathbb{N}) .
$$

It can be written as

$$
T_{\alpha}(f)(x)=\frac{1}{\Gamma(\alpha)} \int_{0}^{\infty} \frac{y^{\alpha-1} f(y)}{(x+y)^{\alpha}} d y, \quad x \in \mathbb{C} \backslash(-\infty, 0] .
$$

Setting $r=\alpha-1, f(t)=y^{\alpha-1} f(y)$ in (2.8), we get

$$
S_{\alpha-1}\left(y^{\alpha-1} f(y)\right)(x)=\int_{0}^{\infty} \frac{y^{\alpha-1} f(y)}{(x+y)^{\alpha}} d y, \quad x \in \mathbb{C} \backslash(-\infty, 0] .
$$

Using (2.10) and (2.11), we obtain the relationship between (2.8) and (2.10),

$$
T_{\alpha}(f)(x)=\frac{1}{\Gamma(\alpha)} S_{\alpha-1}\left(y^{\alpha-1} f(y)\right)(x), \quad x \in \mathbb{C} \backslash(-\infty, 0] .
$$

By changing $x$ by $z$ and $\alpha$ by $r+1$, it follows

$$
\Gamma(r+1) T_{r+1}(f)(z)=S_{r}\left(y^{r} f\right)(z), \quad z \in \mathbb{C} \backslash(-\infty, 0], r \in \mathbb{R} \backslash(-\mathbb{N}) .
$$


2.3. Modified Stieltjes transformation $T_{r+1}$. The modified Stieltjes transformation $T_{r+1}(f), r \in \mathbb{R} \backslash(-\mathbb{N})$, is complex-valued function defined by

$\Gamma(r+1) T_{r+1}(f)(s)=(r+1)_{k} \int_{0}^{\infty} \frac{F(t)}{(s+t)^{r+1+k}} d t, \quad r \in \mathbb{R} \backslash(-\mathbb{N}), s \in \mathbb{C} \backslash(-\infty, 0], 0<t<\infty$,

where $(\alpha)_{k}=(\alpha) \cdot(\alpha+1) \cdot(\alpha+2) \cdots(\alpha+k-1)$.

2.4. Generalized modified Stieltjes transformation $\widetilde{T}_{r+1}$. The $\widetilde{T}_{r+1}$-transformation of a distribution $f \in S_{+}^{\prime}(\mathbb{R})$ is complex-valued function $\widetilde{T}_{r+1}(f)$ defined by

$$
\begin{aligned}
& \Gamma(r+1) \tilde{T}_{r+1}(f)(s) \\
& \quad=\lim _{w \rightarrow \infty}\left\langle f(t), \eta(t)(s+t)^{-r-1} \exp (-w t)\right\rangle, \quad w \in \mathbb{R}, s \in \Lambda \subset(\mathbb{C} \backslash(-\infty, 0]), \eta \in A(s) .
\end{aligned}
$$

Here $\Lambda$ is the set of complex numbers for which this limit exists and $A(s)$ is the family of all smooth functions, defined on $\mathbb{R}$ for which there exists $\varepsilon=\varepsilon_{\eta, s}>o$ such that $0 \leq \eta(t) \leq 1$, $t \in \mathbb{R}, \eta(t)=1$, if $t$ belongs to the $\in$-neighbourhood of $\overline{\mathbb{R}}_{+} \eta(t)=0$ or if it belongs to the complement of the $2 \varepsilon$-neighbourhood of $\overline{\mathbb{R}}_{+}$, where $\varepsilon>0$ is arbitrary if $\operatorname{Im} s \neq 0$, and $0<$ $2 \varepsilon<\max \operatorname{Re} s$, if for some $\operatorname{Im} s=0$ and $\left|D^{p} \eta(t)\right| \leq C_{p}, t \in \mathbb{R}$. If $\eta(t) \in A(s), s \in\left(\mathbb{C} \backslash \mathbb{R}_{-}\right)$, then $\eta(t)(s+t)^{-r-1} \exp (-w, t) \in S(\mathbb{R})$ for $w \in \mathbb{R}_{+}, r \in \mathbb{R}$.

\section{Main results}

3.1. Tauberian-type theorems for modified Stieltjes transformation. For the main results of this section, we need the following assertion from [5].

Theorem 3.1. Suppose that for some $m>0$ and

$$
x \longrightarrow \infty, \quad \int_{0}^{\infty} \frac{d \phi(\lambda)}{(\phi+x)^{m+1}} \sim \int_{0}^{\infty} \frac{d \varphi(\lambda)}{(\phi+x)^{m+1}}
$$

and the following conditions are satisfied.

(1) Functions $\phi$ and $\varphi$ are defined for $x>0$ and are nondecreasing.

(2) $\lim _{x \rightarrow \infty} \phi(x)=\infty$.

(3) For any $C>1$, there are constants $\gamma$ and $\mathbb{N}, 0<\gamma<m, \mathbb{N}>0$, such that for any $x>y>\mathbb{N}, \phi(x) / \phi(y) \leq C(x / y)$. Then for $\lambda \longrightarrow \infty, \phi(\lambda) \sim \varphi(\lambda)$.

(This means $|\phi(\lambda) / \varphi(\lambda)-1|<\varepsilon$ if $\lambda>\lambda_{0}(\varepsilon), \lambda \in B$, meas $\left(\left(\lambda_{0}, \infty\right) \backslash B\right)=0$.) Let us note that condition (3) is called as the Keldysh-type condition.

Now we are ready to prove the first Tauberian result.

Theorem 3.2. Suppose that $s>1, r+m-s>0, f \in L^{\prime}(r)$, and $F($ see (2.6)) is a nondecreasing function. Moreover, let

$$
\Gamma(r+1)\left(T_{r+1} f\right)(x) \sim \frac{\Gamma(s)}{\Gamma(r+1)} \cdot \frac{L(s)}{x^{s}}, \quad x \longrightarrow \infty
$$


where $L$ is a slowly varying function at $\infty$ which is defined in some interval $[A, \infty)$, such that $x^{r=k-s} L(x)$ is a nondecreasing function. Then $f$ has the quasiasymptotic behaviour at $\infty$ related to $k^{r-s} L(k)$ with the limit $C x^{r-s}$, where $C \neq 0$.

Proof. Let us put

$$
\phi(x)= \begin{cases}\frac{x^{r+m-s} L(x)}{\Gamma(r+m-s+1)}, & x>A, \\ 0, & x \leq A .\end{cases}
$$

Then $\phi$ has the quasiasymptotic behaviour at $\infty$ related to $k^{r+m-s} L(k)$ with the limit

$$
f_{r+m-s+1} \text {. }
$$

Hence

$$
\int_{0}^{\infty} \frac{d \phi(t)}{(x+t)^{r+m}}=(r+m) \int_{0}^{\infty} \frac{\phi(t) d t}{(x+t)^{r+m+1}} \sim \frac{(r+m) \Gamma(s)}{\Gamma(r+m+1)} \cdot \frac{L(x)}{x^{s}}, \quad x \longrightarrow \infty .
$$

Now, we show that the conditions of Theorem 3.1 hold for $\phi$ and $F$. In fact, we have only to show that for some $\gamma, 0<\gamma<r+m-1$, and every $C>1$, there exists $\mathbb{N}>0$, such that

$$
\frac{\phi(\lambda y)}{\phi(y)}<C \lambda^{y}, \quad \text { for } \lambda>1, y>\mathbb{N} \text {. }
$$

Let us put $\gamma=r+m-s+\varepsilon$, where we choose $\varepsilon>0$ such that $\gamma>0$ and $\varepsilon<s-1$. After substituting $\phi$ in (3.6), we obtain $L(\lambda y) \leq C \lambda^{\varepsilon} L(y)$ and this inequality is true if $\lambda>1$ and $y>\mathbb{N}$, where $\mathbb{N}$ depends on $C$.

From the assumption that $f \in L^{\prime}(r)$ and from (3.5), we have

$$
\begin{aligned}
\Gamma(r+1)\left(T_{r+1} f\right)(x) & =(r+1)_{m} \int_{0}^{\infty} \frac{F(t)}{(x+t)^{r+m+1}} d t \\
& =(r+1)_{m-1} \int_{0}^{\infty} \frac{d F(t)}{(x+t)^{r+m}} \sim \frac{\Gamma(s)}{\Gamma(r+1)} \cdot \frac{L(x)}{x^{s}} \\
& \sim \int_{0}^{\infty} \frac{d \phi(t)}{(x+t)^{r+m}}, \quad x \rightarrow \infty .
\end{aligned}
$$

This implies that

$$
\Gamma(r+m+1)\left(T_{r+m+1} F\right)(x) \sim \Gamma(r+m+1)\left(T_{r+m+1} \phi\right)(x), \quad x \longrightarrow \infty,
$$

and by Theorem 3.1, it follows that $F \sim \phi, x \rightarrow \infty$.

Thus we obtain that

$$
F(x) \sim \frac{\left(x^{r+m-s} L(x)\right)}{\Gamma(r+m-s+1)}, \quad x \rightarrow \infty
$$


6 Tauberian-type theorems to Stieltjes transformations

Since $r+m-s>0$, it follows that $f$ has the quasiasymptotic behaviour at $\infty$ related to $k^{r+m-s} L(k)$ with the limit $x^{r+m-s}$.

Since $f=t^{-r} D^{m} F$, it easily follows that $f$ has the quasiasymptotic behaviour at $\infty$ related to $k^{r-s} L(k)$ with the limit $C x^{r-s}$, where $C$ is a suitable nonzero constant. This completes the proof of the theorem.

\section{Tauberian-type results related to the quasiasymptotic behaviour}

For the quasiasymptotic behaviour of all original $f$ and for the ordinary asymptotic of the corresponding function $\Gamma(r+1)\left(T_{r+1} f\right)$, we need the following theorem and [5, Lemmas $1,2$, and 3$]$.

Theorem 4.1. Let $a_{n, m}, n, m \in \mathbb{N}$, be a matrix of complex numbers.

(i) If $a_{n, m}$ converges uniformly in $m \in \mathbb{N}$ to $a_{m}$ as $n \rightarrow \infty$ and $\lim _{m \rightarrow \infty} a_{m}$ exists, then

$$
\lim _{n \rightarrow \infty} \lim _{m \rightarrow \infty} a_{n, m}=\lim _{m \rightarrow \infty} \lim _{n \rightarrow \infty} a_{n, m}=\lim _{\substack{n \rightarrow \infty \\ m \rightarrow \infty}} a_{n, m}
$$

(ii) If $\lim _{n \rightarrow \infty} a_{n, m}$ exists for every $n \in \mathbb{N}, \lim _{m \rightarrow \infty} a_{n, m}$ exists for every $n \in \mathbb{N}, \lim _{n, m \rightarrow \infty}$ $a_{n, m}$ exists, then $a_{n, m}$ converges uniformly in $n \in \mathbb{N}$ as $m \rightarrow \infty$.

Lemma 4.2. Let $r \in \mathbb{R} \backslash(-\mathbb{N}), k \in \mathbb{N}_{0}$ be given and let $\gamma \in \mathbb{C}$, then for every $n \in \mathbb{N}$,

$$
\begin{gathered}
\sum_{i=1}^{n+1}\left(\begin{array}{c}
n+1 \\
i
\end{array}\right)(-1)^{i}(2 n+r+k+3) \cdots(2 n+r+k-i)(2 n+r+k+\gamma+3-i) \\
\cdots(r+k+\gamma+i+2)+(2 n+r+k+\gamma+3) \cdots(r+k+\gamma+2) \\
=(-1)^{n} \gamma(1-\gamma) \cdots(n-\gamma)(r+k+\gamma+2)_{(n+1) .}
\end{gathered}
$$

Lemma 4.3. Suppose that $f \in S_{+}^{\prime}$ and that $f$ has the quasiasymptotic at $\infty$ related to $k^{v} L(k)$, where $v<r$. Then there exist $k \in \mathbb{N}_{0}, k+v>0$, and a continuous function $F$, $\operatorname{supp} F \subset[0, \infty)$, such that $f=t^{-r} D^{k} F$ and $F_{1}(x)=\int_{0}^{x} F(t) d t, x \in \mathbb{R},\left(\angle_{n, r, k+1, x} \Gamma(r+k+2)\left(T_{r+k+2} F_{1}\right)(x)-\right.$ $\left.F_{1}(x)\right) /\left(x(1+x)^{\nu+k} L(x)\right)$ converges uniformly to zero in $(0, \infty)$.

Lemma 4.4. Let $f \in L^{\prime}(r)$ and $\Gamma(r+1)\left(T_{r+1} f\right)(x) \sim x^{\nu} L(x), x \rightarrow \infty, v>-1$.

Then $\overline{\Gamma(r+1)\left(T_{r+1} f\right)_{+}}(x)$ has the quasiasymptotic behaviour at $\infty$ related to $k^{\nu} L(k)$.

Proof. We have $(\phi \in S)$,

$$
\begin{aligned}
\frac{1}{k^{\nu} L(k)}\left\langle\overline{\Gamma(r+1)\left(T_{r+1} f\right)_{+}}(k x), \phi(x)\right\rangle= & \frac{1}{k^{v+1} L(k)}\left\langle\overline{\Gamma(r+1)\left(T_{r+1} f\right)_{+}}(x), \phi\left(\frac{x}{k}\right)\right\rangle \\
= & \frac{1}{k^{v+1} L(k)} \int_{0}^{1} \Gamma(r+1)\left(T_{r+1} f\right)(x) \\
& \times\left(\phi\left(\frac{x}{k}\right)-\phi(0)-\cdots-\left(\frac{x}{k}\right)^{l-1} \frac{\phi^{(l-1)}(0)}{(l-1) !}\right) \cdot d x \\
& +\frac{1}{k^{v+1} L(k)} \int_{1}^{\infty} \Gamma(r+1)\left(T_{r+1} f\right)(x) \phi\left(\frac{x}{k}\right) d x .
\end{aligned}
$$


Since the first part on the right-hand side of (4.3) converges to zero as $k \rightarrow \infty$, we have to prove that $\left(1 / k^{\nu} L(k)\right) \int_{1 / k}^{\infty} \Gamma(r+1)\left(T_{r+1} f\right)(k x) \phi(x) d x \rightarrow \int_{0}^{\infty} x^{\nu} L(x) \phi(x) d x$ as $x \rightarrow \infty$.

Let us recall $\Gamma(r+1)\left(T_{r+1} f\right)(x) \sim x^{\nu} L(x), x \rightarrow \infty$.

This implies that for a given $\varepsilon>0$, there exists $x_{0}>0$, such that

$$
\left|\Gamma(r+1)\left(T_{r+1} f\right)(x)-x^{\nu} L(x)\right| \leq \varepsilon x^{\nu} L(x), \quad x \geq x_{0}>1 .
$$

We use the following decomposition:

$$
\begin{aligned}
& \frac{1}{k^{\nu} L(k)} \int_{1 / k}^{\infty} \Gamma(r+1)\left(T_{r+1} f\right)(k x) \phi(x) d x \\
& \quad=\frac{1}{k^{v} L(k)}\left[\begin{array}{c}
\int_{1 / k}^{x_{0} / k} \Gamma(r+1)\left(T_{r+1} f\right)(k x) \phi(x) d x \\
+\int_{x_{0} / k}^{\infty} \Gamma(r+1)\left(T_{r+1} f\right)(k x) \phi(x) d x
\end{array}\right] S .
\end{aligned}
$$

The first member on the right-hand side of (4.5) tends to zero, when $k \rightarrow \infty$, because

$$
\frac{1}{k^{\nu} L(k)} \int_{1 / k}^{x_{0} / k}\left|\Gamma(r+1)\left(T_{r+1} f\right)(k x) \phi(x)\right| d x \leq \frac{M}{k^{\nu} L(k)} \max _{x \in \mathbb{R}}\{|\phi(x)|\} \frac{x_{0}-1}{k},
$$

where $M=\max \left\{\left|\Gamma(r+1)\left(T_{r+1} f\right)(x)\right|: 1 \leq x \leq x_{0}\right\}$. Also, one can prove easily that or a given $x_{0}>1$,

$$
\frac{1}{L(k)} \int_{0}^{x_{0} / k}\left|x^{\nu} L(x) \phi(x)\right| d x \longrightarrow 0 \quad \text { as } k \longrightarrow \infty .
$$

Now by (4.4), (4.6), (4.7), we have

$$
\begin{aligned}
& \left|\frac{1}{k^{v} L(k)} \int_{1 / k}^{\infty} \Gamma(r+1)\left(T_{r+1} f\right)(k x) \phi(x) d x-\frac{1}{k^{\nu} L(k)} \int_{0}^{\infty}(k x)^{v} L(k x) \phi(x) d x\right| \\
& \leq \frac{1}{k^{v} L(k)} \int_{1 / k}^{x_{0} / k}\left|\Gamma(r+1)\left(T_{r+1} f\right)(k x) \phi(x)\right| d x+\frac{1}{k^{\nu} L(k)} \int_{0}^{x_{0} / k}\left|(k x)^{v} L(k x) \phi(x) d x\right| \\
& \quad+\frac{1}{k^{v} L(k)} \int_{x_{0} / k}^{\infty}\left|\Gamma(r+1)\left(T_{r+1} f\right)(k x)-(k x)^{v} L(k x)\right||\phi(x)| d x .
\end{aligned}
$$

Now we completed the proof of the lemma.

Now we will assume that $L$ satisfies the inequality $L(m x) / L(m) \leq C(1+x), x>0, m>0$.

Now we are ready to prove the following.

THeOREM 4.5. If $f \in S_{+}^{\prime}$ and $f$ has the quasiasymptotic behaviour at $\infty$ related to $k^{\nu} L(k)$, $r-1<v<r, r \in \mathbb{R} \backslash(-\mathbb{N})$, then the double sequence

$$
\left\langle\frac{\overline{\Gamma(r+k+2)\left(T_{r+k+2} F_{1}\right)_{+}}(x m)}{m^{v+k+1} L(m)}, \angle_{n, r, k+1} \phi^{(k+1)}(x)\right\rangle, \quad m, n \in \mathbb{N}, \phi \in s,
$$


8 Tauberian-type theorems to Stieltjes transformations

converges uniformly in $n \in \mathbb{N}$ as $m \rightarrow \infty$, where $k \in \mathbb{N}, k+v>0$, and $F_{1}$ are defined in Lemma 4.4 .

Proof. Let us put

$$
a_{n, m}=\left\langle\frac{\tilde{\Sigma}_{n, r, k, x} \overline{\Gamma(r+1)\left(T_{r+1} f\right)_{+}}(m x)}{m^{\nu} L(m)}, \phi(x)\right\rangle .
$$

Since

$$
a_{n, m}=(-1)^{k+1}(r+1)_{k+1}\left\langle\frac{\overline{\Gamma(r+k+2)\left(T_{r+k+2} F_{1}\right)_{+}}(m x)}{m^{\nu+k+1} L(m)}, L_{n, r, k+1, x} \phi^{(k+1)}(x)\right\rangle,
$$

we have to prove that $a_{n, m}$ converges uniformly in $n \in \mathbb{N}$ as $m \rightarrow \infty$.

First, we will prove that the conditions of Theorem 4.1(i) are satisfied. Then, from Theorem 4.1(ii), the assertion of this theorem will follow

$$
a_{n, m} \longrightarrow a_{m}=\left\langle\frac{f(m x)}{m^{v} L(m)}, \phi(x)\right\rangle, \quad n \longrightarrow \infty, m \in \mathbb{N}
$$

Since

$$
\begin{aligned}
a_{n, m}-a_{m}=(-1)^{k+1}(r+1)_{k+1} \int_{0}^{\infty} \frac{L_{n, r, k+1, x} \Gamma(r+k+2)\left(T_{r+k+2} F_{1}\right)(m x)}{m^{v+k+1} L(m)} \phi^{(k+1)}(x) d x \\
=(-1)^{k+1}(r+1)_{k+1} \int_{0}^{\infty} \frac{L_{n, r, k+1, x} \Gamma(r+k+2)\left(T_{r+k+2} F_{1}\right)(m x)-F_{1}(m x)(m x)}{(m x)(1+m x)^{v+k}} \\
\times \frac{(1+m x)^{\nu+k} L(m x)}{m^{v+k+1} L(m)} \phi^{(k+1)}(x) d x,
\end{aligned}
$$

from Lemma 4.4 and the fact that

$$
\frac{(m x)(1+m x)^{\nu+k} L(m x)}{m^{\nu+k+1} L(m)} \leq 2 C(1+x) \frac{m x\left(1+(m x)^{\nu+k}\right)}{m^{\nu+k+1}} \leq 2 C(1+x)\left(x+x^{\nu+k+1}\right), \quad x \geq 0,
$$

we obtain that $a_{n, m}-a_{m} \rightarrow 0$ uniformly in $n \in \mathbb{N}$ as $n \rightarrow \infty$.

We have $\Gamma(r+1)\left(T_{r+1} f\right)(x) \sim(\Gamma(r-v) / \Gamma(r+1)) x^{\nu-r+2} L(x), x \rightarrow \infty$. Since $-1<v-$ $r$, we have by Lemma 4.4 that $\overline{\Gamma(r+1)\left(T_{r+1} f\right)_{+}}$has the quasiasymptotic at $\infty$ related to $k^{\nu-r} L(k)$ with the limit $(\Gamma(r-v) / \Gamma(r+1)) x^{\nu-r}$. 
By Leibniz formula, we have

$$
\begin{aligned}
& D^{n+1} x^{2 n+r+k+3} D^{n+1} \Gamma(r+k+2)\left(T_{r+k+2} F_{1}\right)(x) \\
& =D^{n+1} \sum_{i=0}^{n+1}(-1)^{i}\left(\begin{array}{c}
n+1 \\
i
\end{array}\right)\left(\left(x^{2 n+r+k+3}\right)^{(i)} \Gamma(r+k+2)\left(T_{r+k+2} F_{1}\right)(x)\right)^{(n+1-i)} \\
& =\sum_{i=1}^{n+1}(-1)^{i}\left(\begin{array}{c}
n+1 \\
i
\end{array}\right)(2 n+r+k+3) \cdots(2 n+r+k+4-i) \\
& \quad \times\left(\left(x^{2 n+r+k+3-i}\right)\left(\Gamma(r+k+2)\left(T_{r+k+2} F_{1}\right)(x)\right)^{(2 n+2-i)}\right. \\
& \left.+\left(x^{2 n+r+k+3}\right) \Gamma(r+k+3)\left(T_{r+k+3} F_{1}\right)(x)\right)^{(2 n+2)} .
\end{aligned}
$$

Let $\gamma=v-r$. Then $x^{2 n+r+k+3-i} \Gamma(r+k+2)\left(T_{r+k+2} F_{1}\right)(x)$ has the quasiasymptotic behaviour at $\infty$ related to $k^{2 n+r+k+\gamma+3-i} L(k)$ with the limit $\Gamma(-\gamma) x^{2 n+r+k+\gamma+3-i} / \Gamma(r+1)(r+1)_{k+1}$.

Lemma 4.2 and the properties of quasiasymptotic behaviour imply that $D^{n+1}$ $x^{2 n+r+k+3} D^{n+1} \Gamma(r+k+2)\left(T_{r+k+2} F_{1}\right)(x)$ has the quasiasymptotic behaviour at $\infty$ related to $k^{r+k+\gamma+1} L(k)$ with the limit

$$
(-1)^{n} \gamma(1-\gamma) \cdots(n-\gamma)(r+k+\gamma+2)_{n+1} \frac{\Gamma(-\gamma)}{\Gamma(r+1)(r+1)_{k+1}} x^{\gamma+k+r+1} .
$$

Thus, we obtain

$$
\begin{aligned}
\lim _{n \rightarrow \infty} \lim _{m \rightarrow \infty}(-1)^{(k+1)}(r+1)_{k+1}\left\langle\frac{\angle_{n, r, k+1, x} \Gamma(r+k+2)\left(T_{r+k+2} F_{1}\right)_{+}(m x)}{m^{\nu+k+1} L(m)}, \phi(x)\right\rangle \\
=-\lim _{n \rightarrow \infty} \frac{(-1)^{k+1} \Gamma(r+k+2)}{(n+1) ! \Gamma(n+r+k+2)} \frac{\Gamma(n-\gamma+1)}{\Gamma(-\gamma)} \frac{\Gamma(r+k+\gamma+n+3)}{\Gamma(r+k+\gamma+2)} \frac{\Gamma(-\gamma)}{\Gamma(r+1)} \\
\quad \times\left\langle x^{\nu+k+1}, \phi^{(k+1)}(x)\right\rangle .
\end{aligned}
$$

To prove that the last limit exists, we have to use the Stirling formula.

$\Gamma(s+1) \sim \sqrt{2 \pi} e^{-s} s^{(s+1) / 2}, s \rightarrow \infty$. Thus for the double sequence $a_{n, m}$, Theorem 4.1(i) holds and Theorem 4.1(ii) implies the assertion.

Theorem 4.6. Let $f \in L^{\prime}(r)$ and let $\overline{\Gamma(r+1)\left(T_{r+1} f\right)_{+}}(x)$ have the quasiasymptotic at $\infty$ related to $k^{\alpha} L(k),-1<\alpha<0$. If for any $\phi \in s$, the double sequence (4.9) converges uniformly in $n \in \mathbb{N}$ as $m \rightarrow \infty$, then $f$ has the quasiasymptotic at $\infty$ related to $k^{\alpha+r} L(k)$.

Proof. If $a_{n, m}$ is the double sequence defined in the proof of Theorem 4.5, we have by Theorem 4.1(i) the assertion.

\section{Acknowledgments}

The first author is thankful to the referees for their valuable suggestions. 


\section{Tauberian-type theorems to Stieltjes transformations}

\section{References}

[1] J. N. Drožžinov and B. I. Zavialov, Quasiasymptotic behavior of generalized functions, and Tauberian theorems in the complex domain, Matematicheskii-Sbornik.-Novaya-Seriya 102(144) (1977), no. 3, 372-390.

[2] J. Lavoine and O. P. Misra, Abelian theorems for the distributional Stieltjes transformation, Mathematical Proceedings of the Cambridge Philosophical Society 86 (1979), no. 2, 287-293.

[3] O. I. Marichev, Handbook of Integral Transforms of Higher Transcendental Functions. Theory and Algorithmic Tables, Ellis Horwood Series: Mathematics and Its Applications, Ellis Horwood, Chichester; John Wiley \& Sons, New York, 1983.

[4] S. Pilipović, An inversion theorem for the Stieltjes transform of distributions, Proceedings of the Edinburgh Mathematical Society. Series II 29 (1986), no. 2, 171-185.

[5] S. Pilipović, B. Stanković, and A. Takači, Asymptotic Behaviour and Stieltjes Transformation of Distributions, Teubner-Texte zur Mathematik, vol. 116, BSB B. G. Teubner Verlagsgesellschaft, Leipzig, 1990.

[6] E. Seneta, Regularly Varying Functions, Lecture Notes in Math, vol. 508, Springer, Berlin, 1976.

[7] B. Stanković, Abelian and Tauberian theorems for the Stieltjes transform of distributions, Russian Mathematical Surveys 40 (1985), no. 4, 99-113.

[8] B. I. Zavialov, Y. N. Drozinov, and V. S. Vladimirov, Several Dimension Tauberian Theorems for Generalized Functions in Mathematical Physics, Nauka, Moscow, 1986.

S. B. Gaikwad: Department of Mathematics, New Arts, Commerce and Science College Parner, Pune University, Pune, District Ahmednagar 414302, Maharashtra, India

E-mail address: shrigaik@yahoo.com

M. S. Chaudhary: Department of Mathematics, Shivaji University, Vidayanagar, Kolhapur 416004, Maharashtra, India

E-mail address: m_s_chaudhary@rediffmail.com 


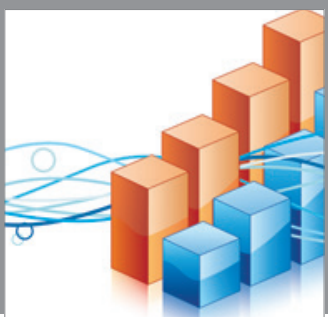

Advances in

Operations Research

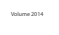

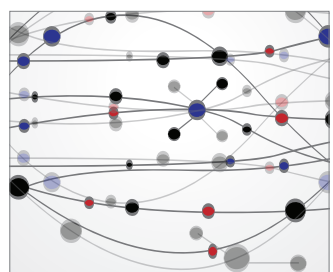

\section{The Scientific} World Journal
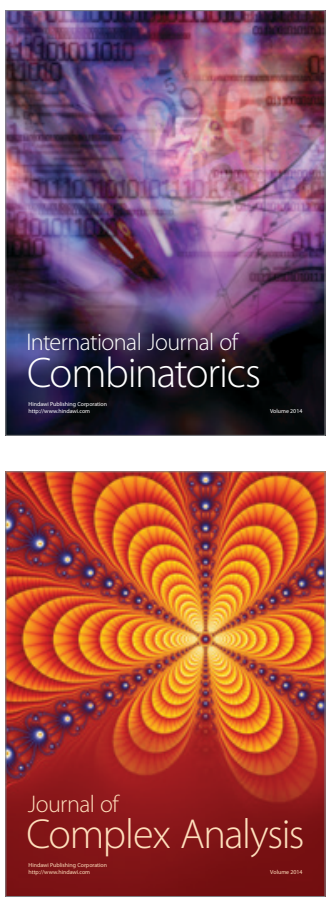

International Journal of

Mathematics and

Mathematical

Sciences
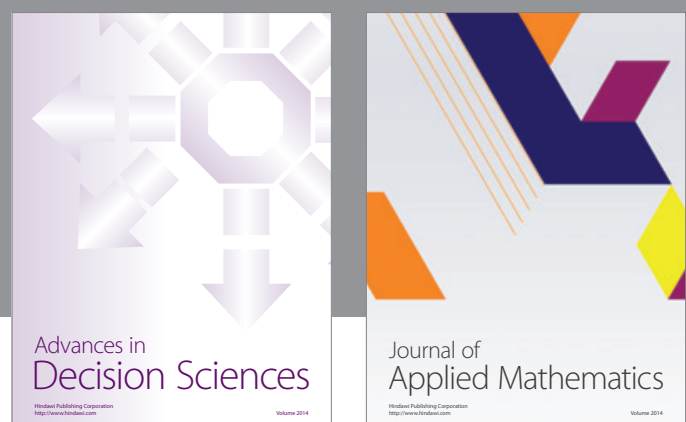

Journal of

Applied Mathematics
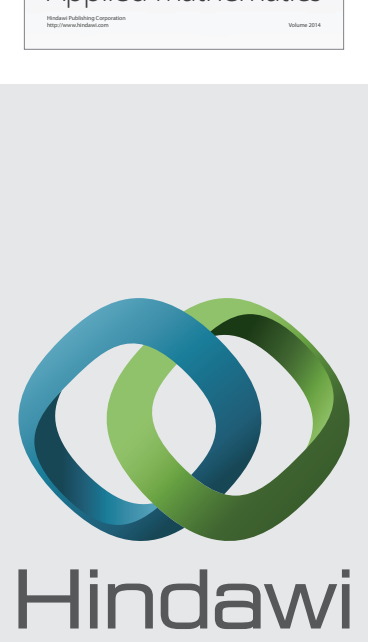

Submit your manuscripts at http://www.hindawi.com
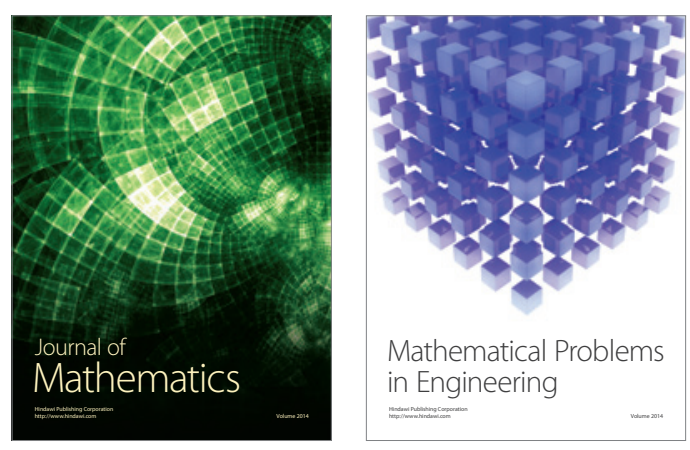

Mathematical Problems in Engineering
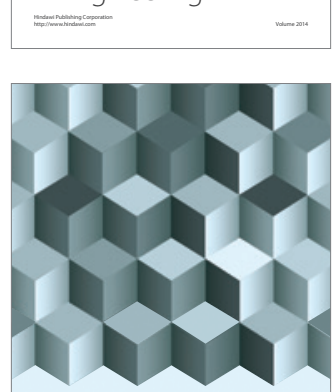

Journal of

Function Spaces
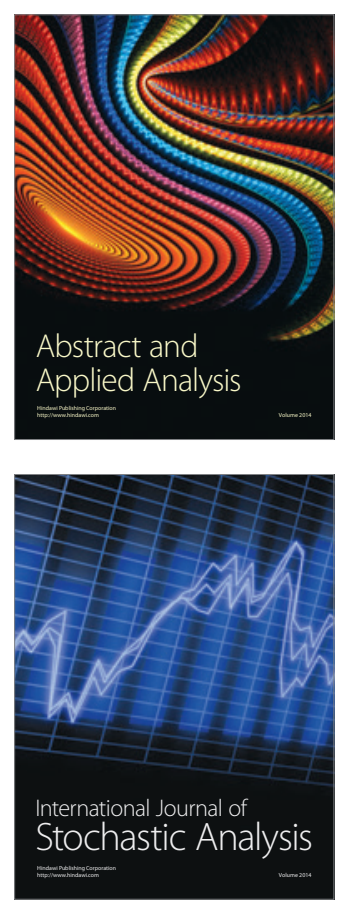

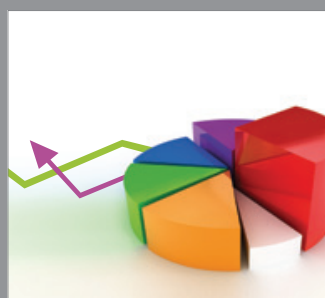

ournal of

Probability and Statistics

Promensencen
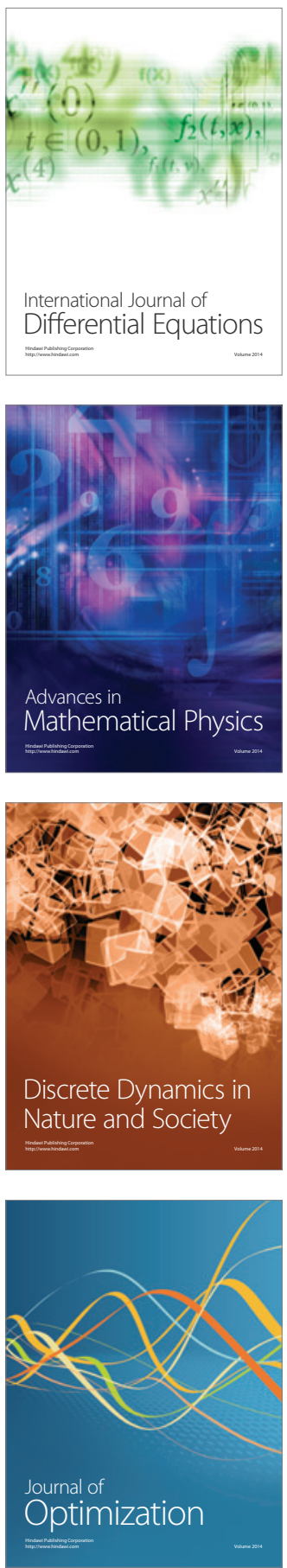